\title{
Editorial
}

\section{Recent Advances in Mobile Cloud Computing}

\author{
Dharma P. Agrawal $\mathbb{D},{ }^{1}$ Brij B. Gupta, ${ }^{2}$ Shingo Yamaguchi, ${ }^{3}$ and Konstantinos E. Psannis ${ }^{4}$ \\ ${ }^{1}$ Center for Distributed and Mobile Computing, EECS Department, University of Cincinnati, Cincinnati, OH 45221-0030, USA \\ ${ }^{2}$ Department of Computer Engineering, National Institute of Technology, Kurukshetra 136119, India \\ ${ }^{3}$ Yamaguchi University, Yamaguchi, Japan \\ ${ }^{4}$ University of Macedonia, Thessaloniki, Greece
}

Correspondence should be addressed to Dharma P. Agrawal; dharmaagrawal@gmail.com

Received 15 February 2018; Accepted 18 February 2018; Published 22 March 2018

Copyright (C) 2018 Dharma P. Agrawal et al. This is an open access article distributed under the Creative Commons Attribution License, which permits unrestricted use, distribution, and reproduction in any medium, provided the original work is properly cited.

Cloud computing has become increasingly important due to many associated characteristics such as low cost storage, availability of data at any time and any place, and ease in maintenance. Mobile devices can collect personal data from various sensors within a shorter period of time and sensorbased data consists of valuable information from users. But, mobile access introduces many problems such as duplication to make data easily accessible, access to desired data, security of data, and AI techniques for quick and effective access to data. In this special issue on recent advances in mobile cloud computing, we have invited a few papers that address such issues.

The paper titled "Workflow Scheduling Using Hybrid GA-PSO Algorithm in Cloud Computing" introduces a hybrid algorithm that efficiently allocates tasks to the resources so as to balance the system load that leads to reduction in execution cost with faster convergence. Migrating processor-intensive tasks to resource-rich surrogates has been limited due to limited practical settings and a new novel self-contained compatible code framework has been designed in the paper titled "MobiCOP: A Scalable and Reliable Mobile Code Offloading Solution." The paper titled "Quality of Experience Assessment of Video Quality in Social Clouds" compares compression of video data using three popular social clouds and present achieved video quality.

The paper titled "Context-Aware Cloud Service Selection Model for Mobile Cloud Computing Environments" considers appropriate service selection model for Mobile Cloud system using ns-3 simulator considering user's trust expectation. Indexing of database has been used for efficient query processing that makes index excessively large for voluminous data and has been addressed in the paper titled "An Indexing Method to Construct Unbalanced Layers for High-Dimensional Data in Mobile Environments" by constructing a new concept of layer-based index, dividing the dimension of the input data, and computing the convex hull in each dimension. An uneven distribution of the packets could create potential traffic hotspots and could drastically degrade network performance of data center; the paper titled "Layout-Independent Wireless Facility Constructing and Scheduling for Data Center Networks" shows how to efficiently construct and schedule the wireless facilities and experimental results using NS-3 based on two typical data center layouts to validate scheme's effectiveness.

As any unauthorized access to mobile cloud may cause serious security and privacy problems, the paper titled "Secrecy Dimming Capacity in Multi-LED PAM-Based Visible Light Communications" addresses the secrecy dimming capacity of secure transmission over visible light communication channels and could serve as a guideline for practical mobile networks. The paper titled "A Variable Impacts Measurement in Random Forest for Mobile Cloud Computing" considers not only data classification but also data interpretation to gain insight into streaming data using game theory technique. In this way, the influence of variables is analyzed and data interpretation in a random forest can be applied in mobile cloud computing environment.

Dharma P. Agrawal Brij B. Gupta

Shingo Yamaguchi Konstantinos E. Psannis 


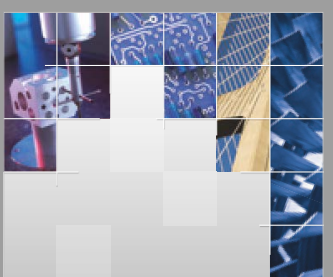

\section{Enfincering}
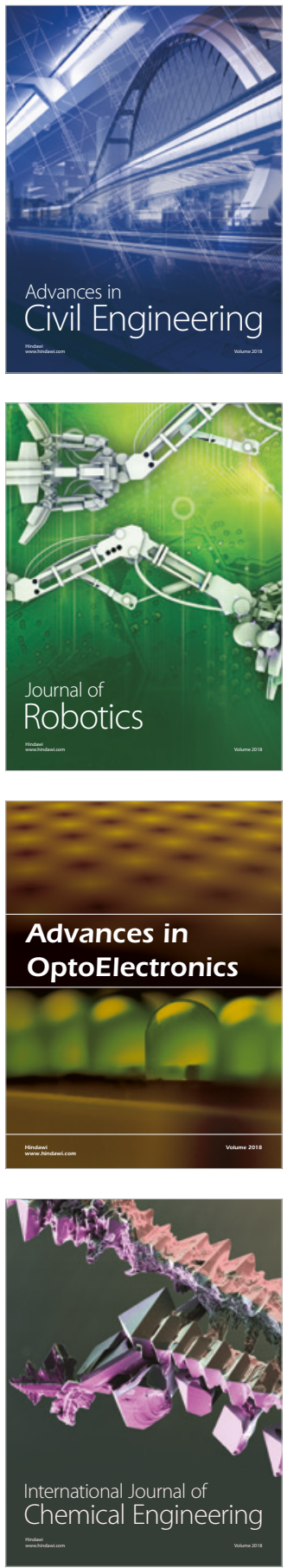

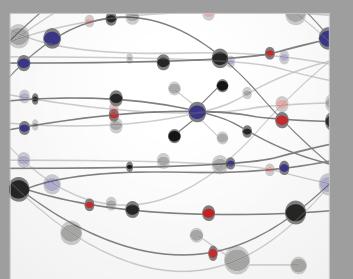

\section{Rotating \\ Machinery}

The Scientific World Journal

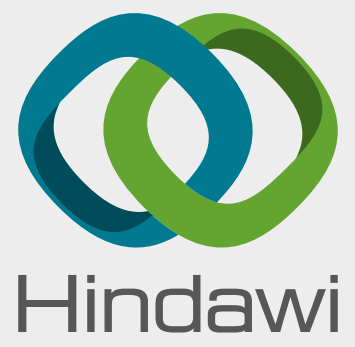

Submit your manuscripts at

www.hindawi.com
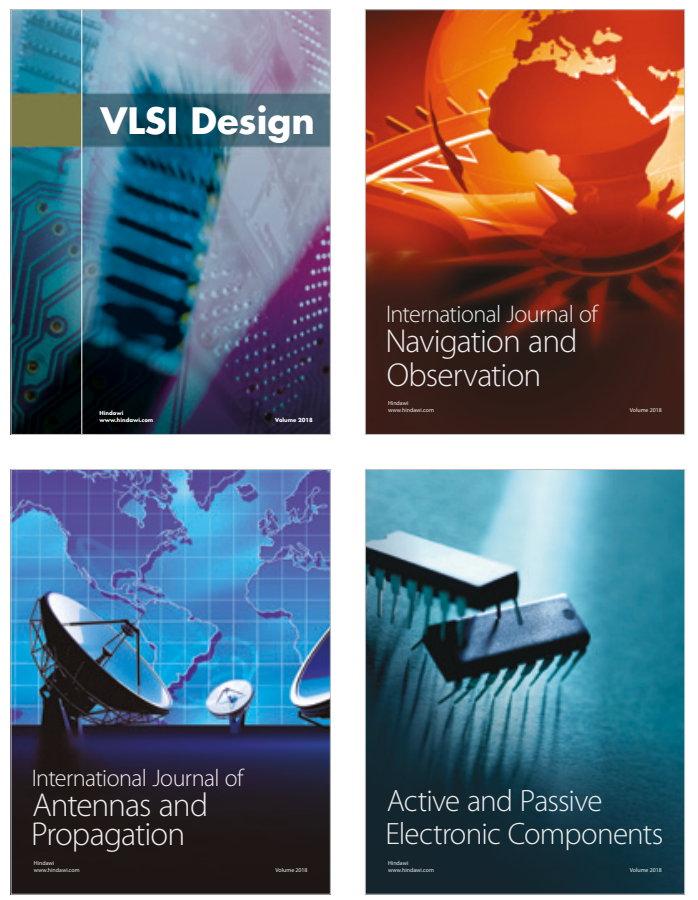
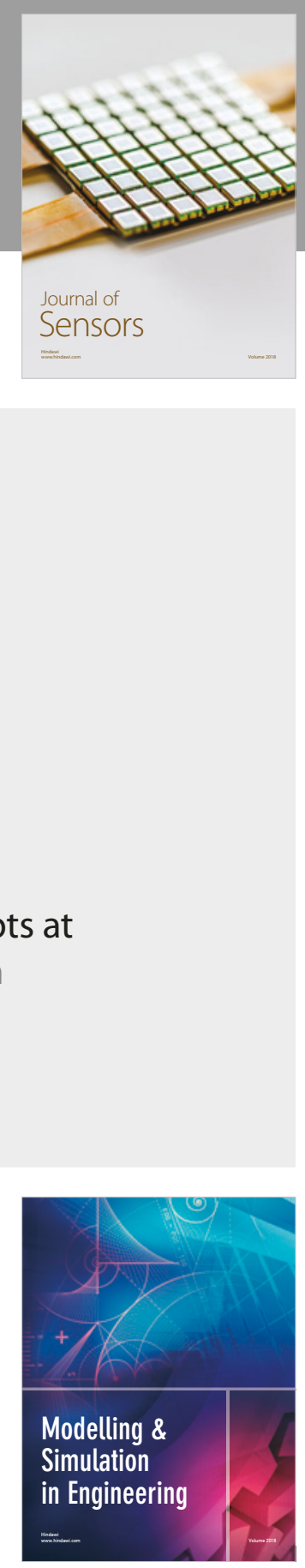

\section{Advances \\ Multimedia}
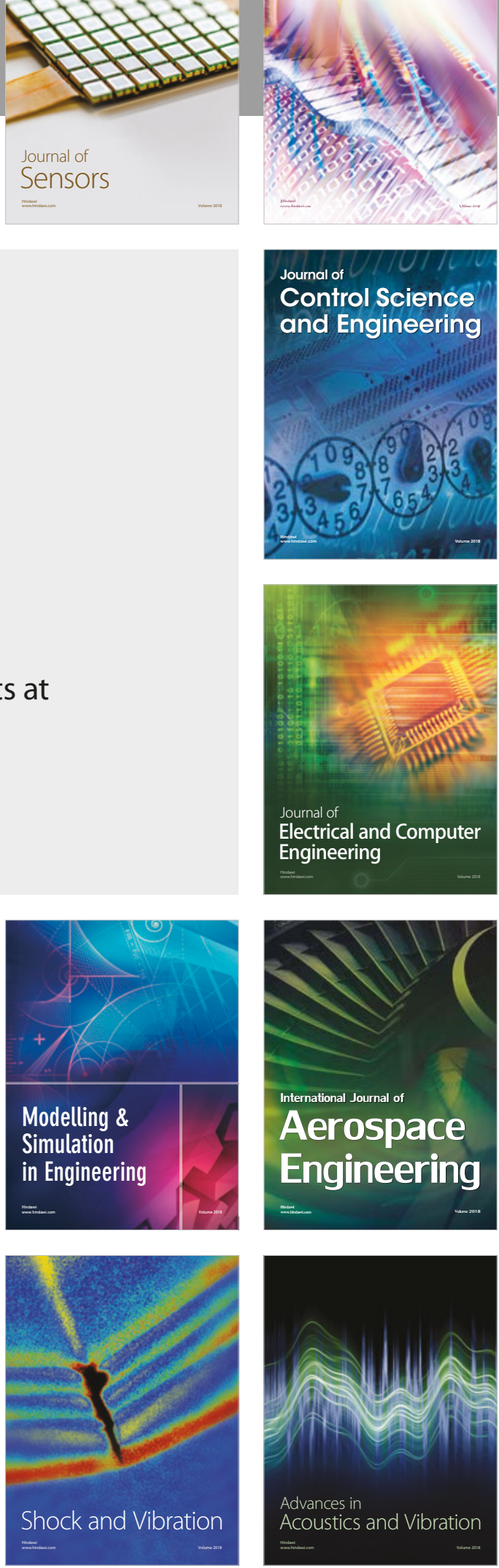